\title{
Rauchen erhöht Risiko für Etagenwechsel
}

\author{
Rauchen spielt eine wichtige Rolle bei der Entstehung von aller- \\ gischen Erkrankungen. Eine Studie untersuchte, welchen Zusammen- \\ hang es zwischen Zigarettenkonsum und der Entwicklung eines \\ Asthma bronchiale bei Patienten mit allergischer Rhinitis gibt?
}

igarettenrauchen fördert die Entwicklung einer chronischen Rhinitis. Diese ist wiederum ein Risikofaktor für ein allergisches Bronchialasthma. Welchen Stellenwert beim Etagenwechsel das Rauchen hat, überprüfte ein italienisches Autorenteam. In einer Langzeitstudie wurden Patienten im Alter zwischen 18 und 40 Jahren beobachtet. Sie zeigten alle bei Studienbeginn typische Rhinokonjunktivitissymptome bei nasaler Provokation mit Allergenen, die zuvor im Pricktest zu einer klaren Reaktion geführt hatten. Rund zehn Jahre nach Studienbeginn wurden die Patienten erneut befragt und untersucht. Insgesamt 325 Teilnehmer beendeten die Studie.

Das Risiko, ein Asthma bronchiale zu entwickeln, war bei Rauchern signifikant um knapp das Dreifache erhöht (Odds Ratio 2,67 bei einfacher Varianzanalyse, 2,98 bei Multivarianzanalyse). Dabei zeigte sich auch eine klare Abhän- gigkeit von der Menge der Zigaretten: Betrug die Zahl der "Packungsjahre“ (Zahl der pro Tag konsumierten Zigaretten mal Jahre, in denen geraucht wurde,

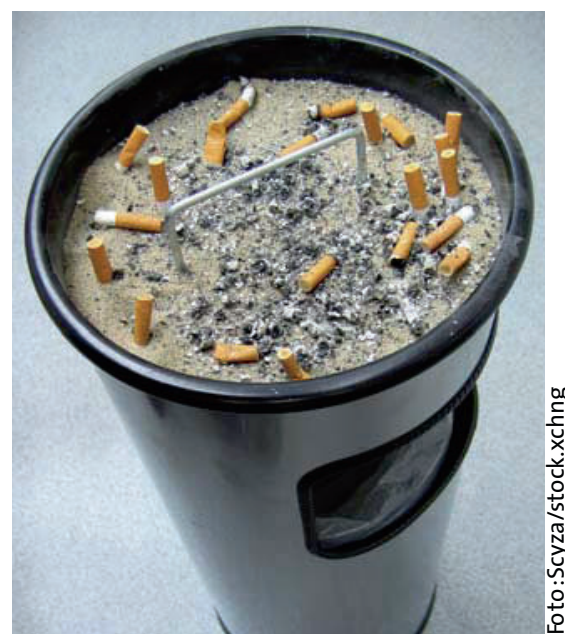

Wer seine letzte Zigarette ausdrückt, reduziert damit auch sein Asthmarisiko. dividiert durch 20) zwischen einem und zehn Jahren, war das Asthmarisiko gegenüber Nichtrauchern um den Faktor 2,05 erhöht. Bei 11 bis 20 Packungsjahren nahm das Risiko um den Faktor 3,71 zu. Noch stärkere Raucher mussten mit einer 5,05-fachen Wahrscheinlichkeit rechnen, Asthma zu entwickeln.

Das Asthmarisiko von ehemaligen Rauchern war gegenüber Nichtrauchern knapp um das Zweifache erhöht, jedoch nur als statistischer Trend. Zusätzliche signifikante Faktoren, die zu einem Asthma bronchiale beitrugen, waren Verschlechterung der Rhinitissymptome, positive Atopieanamnese in der Familie und Kontakt zu Haustieren.

Fazit: Rauchen erhöht bei Patienten mit allergischer Rhinitis das Risiko, ein Asthma bronchiale zu entwickeln, um das Mehrfache. Dabei zeigte sich auch eine signifikante Beziehung zwischen der Menge der konsumierten Zigaretten und der Wahrscheinlichkeit für das Entstehen eines Asthmas.

Polosa R et al. Cigarette smoking is associated with a greater risk of incident asthma in allergic rhinitis. J Allergy Clin Immunol 2008; 121: 1428-34

\section{Schwere Rhinitis - schweres Asthma?}

\section{Asthma und Rhinitis sind miteinander auftretende Erkrankungen. Häufig korreliert der Schweregrad beider Erkrankungen. Konsistent sind die meist retrospektiv erhobenen Daten allerdings nicht.}

Z ur genaueren Untersuchung der Zusammenhänge zwischen den Schweregraden von Asthma bronchiale und allergischer Rhinitis wurden 557 Patienten mit schwerem Asthma in eine prospektive Kohortenstudie einbezogen. Die Studie fand in der brasilianischen Stadt Salvador statt, die meisten Patienten waren zu Studienbeginn ohne regelmäßige Asthmamedikation. Ein Jahr lang wurden alle drei Monate die Notaufnahmen der Studienteilnehmer aufgrund einer Asthmaexazerbation und die Verordnung von topischen Glukokortikoiden zur Asthma- und Rhinitisbehandlung erfasst. Am Ende der Beob- achtungszeit wurden die Patienten auf das Vorliegen einer atopischen bzw. nicht atopischen Rhinitis und deren Schweregrad entsprechend den ARIAKriterien untersucht. Außerdem sollten die Patienten Fragebögen zur Asthmasymptomatik und zur Lebensqualität beantworten.

Von den 557 Asthmapatienten hatten 82 (15\%) keine Rhinitis, 299 (54\%) eine leichte und 176 (31\%) ein mäßige bis schwere Rhinitis. Bei der Auswertung erwies sich eine mäßige/schwere Rhinitis als Prädiktor für Notaufnahmen (Odds Ratio [OR] 3,83) sowie als Prädiktor für ein unkontrolliertes Asthma nach einem
Jahr (OR 12,68). Gleiches galt für eine weniger als 10\%ige Besserung der Atemwegsobstruktion (OR 2,94) und für einen um weniger als 50\%igen Rückgang der Zahl der Notaufnahmen (OR 2,90). Die Schwere der Rhinitis war positiv mit dem Asthmaschweregrad und negativ mit der Lebensqualität korreliert.

Fazit: Bei Patienten mit schwerem Asthma ist eine begleitende mäßige bis schwere Rhinitis ein starker Prädiktor für ein besonders schweres Asthma und ein schlechteres Ansprechen auf die Asthmamedikation. Es sollte nun untersucht werden, ob Patienten mit einem schweren Asthma von der Behandlung der Rhinitis profitieren.

Ponte EV et al. Lack of control of severe asthma is associated with co-existence of moderate-to-severe rhinitis. Allergy 2008; 63: 564-9 